\title{
Domain-Decomposition Approach to Krylov Subspace Iteration
}

\author{
O. A. Iupikov, Student Member, IEEE, C. Craeye, Senior Member, IEEE, R. Maaskant, Senior Member, IEEE, \\ and M. V. Ivashina, Senior Member, IEEE
}

\begin{abstract}
Krylov subspace iterative techniques consist of finding the solution of a scattering problem as a linear combination of "generating vectors" obtained through successive matrix-vector multiplications. This paper extends this approach to domaindecomposition. Here, on each subdomain a subspace is obtained by constructing the segments of each generating vector associated with the subdomain, and by weighting these segments independently, which provides more degrees of freedom. The method is tested for scattering by a sphere and a rectangular plate, as well as radiation from connected arrays with strongly coupled antenna elements. It is shown that substantial computational savings can be obtained for the sphere and the array. This opens up new perspectives for faster solutions of multi-scaled problems.
\end{abstract}

Index Terms-domain-decomposition, CBFM, GMRES, Krylov subspace iteration, connected arrays

\section{INTRODUCTION}

C ONVENTIONAL iterative techniques, such as the Full Orthogonalization Method (FOM) or the Generalized Minimal Residual Method (GMRES) [1], have proved their capability and efficiency to solve large-scale electromagnetic problems. They define a set of current distributions on the whole domain through successive matrix-vector multiplications (mat-vecs) and then solve for their expansion coefficients in an iterative manner. However, these methods become computationally expensive for a large number of generating vectors. To avoid this, a restart procedure is often used, which in addition helps to improve the condition number of the generated system of equations, thereby improving the accuracy of the method.

Many improvements on the GMRES method can be found in the literature. For example, in [2] an adaptive deflation strategy is proposed, which retains useful information at the time of a restart to avoid stagnation and improve the convergence rate.

The generating vectors in GMRES can also be seen as Macro Basis Functions (MBFs) [3], [4]. A similar approach is used in domain-decomposition methods like the Characteristic Basis Functions Method (CBFM) [5] and the Synthetic Functions method (SFX) [6]. A major difference between them is that MBFs in GMRES (or FOM) are defined on the whole

O. A. Iupikov, R. Maaskant, and M. V. Ivashina are with the Signals and Systems Department of the Chalmers University of Technology, Gothenburg, Sweden, e-mail: oleg.iupikov@ chalmers.se,rob.maaskant@ chalmers.se, marianna.ivashina@chalmers.se. C. Craeye is with the Institute of Communication Technologies Electronics and Applied Mathematics (ICTEAM) of the Université catholique de Louvain, Louvain-la-Neuve, Belgium, e-mail: christophe.craeye@uclouvain.be.

This work is financed by Swedish VR and VINNOVA grants, as well as the travel grant from Ericsson's Research Foundation.

Manuscript received April xx, 20xx; revised January xx, 20xx. computational domain and belong to a Krylov subspace, while CBFM-like techniques split the structure into subdomains and analyze them in isolation through the definition of set of independent MBFs on each subdomain, obtained by exciting the subdomain in various ways $^{1}$. Assuming that MBFs are obtained using a multiple-scattering (between subdomains) methodology, a "rule of thumb" is proposed in [7] stating that both FOM and CBFM provide a similar accuracy when the number of iterations in FOM is equal to the average number of MBFs per subdomain in CBFM. However, in some cases, the CBFM yields better accuracy, owing to the fact that it provides more degrees of freedom (DoFs).

In this paper we propose a domain-decomposition approach to Krylov subspace iteration, where MBFs (or CBFs) on each subdomain are naturally constructed from the different segments of the generating vectors.

The paper is organized as follows. In Sec. II we formulate a reduced system of equations built from segments of the generating vectors as MBFs, while avoiding extra mat-vecs. Next, an algorithm for the restart procedure is described in Sec. III. The proposed approach is validated in Sec. IV for the case of a perfectly conducting sphere, a rectangular plate, and an array of electrically connected and disconnected taperedslot antennas. A discussion about complexity and accuracy of the approach follows in Sec. V and conclusions are drawn in Sec. VI.

\section{Segmented Krylov subspace as MBFs}

Consider the Method of Moments (MoM) matrix equation:

$$
\mathbf{Z I}=\mathbf{e}
$$

where $\mathbf{Z}$ is the $N \times N$ MoM matrix; e is the $N \times 1$ excitation vector and $\mathbf{I}$ is a vector containing the expansion coefficients for the elementary basis functions. Accordingly, the reduced CBFM system of equations can be written as

$$
\begin{gathered}
\tilde{\mathbf{Z}} \tilde{\mathbf{i}}=\tilde{\mathbf{e}}, \\
\tilde{\mathbf{Z}}_{i, j}=\mathbf{K}_{i}^{H} \mathbf{Z}_{i, j} \mathbf{K}_{j}, \\
\tilde{\mathbf{e}}_{i}=\mathbf{K}_{i}^{H} \mathbf{e}_{i},
\end{gathered}
$$

where $i, j=1 \ldots M$ are sub-domain indices; $H$ is the Hermitian operator and $\mathbf{K}_{i}$ is the set of MBFs. The method proposed here consists of selecting as MBFs on a given

\footnotetext{
${ }^{1}$ More about the relationship between CBFM-like approaches and Krylov subspace iterative methods can be found in [7].
} 
subdomain the corresponding segments of the generating vectors. Those segments correspond to entries associated to basis functions defined on the subdomain of interest. Hence, the newly proposed MBF selection reads:

$$
\mathbf{K}_{i}=\left[\mathbf{k}_{i}^{(1)}=\mathbf{e}_{i}\left|\mathbf{k}_{i}^{(2)}\right| \ldots \mid \mathbf{k}_{i}^{(P)}\right]
$$

in which generating vector $\mathbf{k}$ is formed iteratively as

$$
\mathbf{k}^{(p+1)}=\mathbf{Z k}^{(p)} \text { for } \quad p=1 \ldots P-1,
$$

where index $i$ refers to the MBF vector entries related to subdomain $i$.

It is important to point out that the most computationally expensive part of the MoM matrix reduction (2b), namely the matrix-matrix product $\mathbf{Z}_{i, j} \mathbf{K}_{j}$, can be carried out during the subspace construction (4). For this purpose, (4) is built from $M^{2}$ smaller matrix-vector products resulting in the $M^{2}$ vectors $\mathbf{v}_{i, j}^{(p)}$, expressed as

$$
\mathbf{v}_{i, j}^{(p)}=\mathbf{Z}_{i, j} \mathbf{k}_{j}^{(p)}
$$

Segment $i$ of the vector $\mathbf{k}^{(p+1)}$ (at the next iteration) is obtained by a simple summation of vectors $\mathbf{v}_{i, j}^{(p)}$ as

$$
\mathbf{k}_{i}^{(p+1)}=\sum_{j} \mathbf{v}_{i, j}^{(p)} .
$$

If the vectors $\mathbf{v}_{i, j}^{(p)}$ are concatenated in a matrix $\mathbf{Q}$ as

$$
\mathbf{Q}_{i, j}=\left[\mathbf{v}_{i, j}^{(1)}\left|\mathbf{v}_{i, j}^{(2)}\right| \ldots \mid \mathbf{v}_{i, j}^{(p)}\right],
$$

then the MoM matrix reduction (2b) can be rewritten as

$$
\tilde{\mathbf{Z}}_{i, j}=\mathbf{K}_{i}^{H} \mathbf{Z}_{i, j} \mathbf{K}_{j}=\mathbf{K}_{i}^{H} \mathbf{Q}_{i, j}
$$

which allows one to reduce by a factor close to two the time involved in (2)-(4), as compared to a straight-forward implementation. The appendix explains how (8) can be modified when the set of MBFs needs to be orthogonalized.

\section{CBFM WITH RESTARTS}

The accuracy of the CBFM method can be significantly improved down to machine precision by introducing a restart procedure similar to that used in a restarted GMRES method [1]:

- Step 1. Initialize the final solution $\mathbf{I}_{\text {fin }}=\mathbf{0}$.

- Step 2. Set the excitation vector e in (2c) to the initial excitation vector $\mathbf{e}_{0}$.

- Step 3. Build and solve the reduced system of equations (2a), compute the solution $\mathbf{I}_{j}=\mathbf{K}_{j} \tilde{\mathbf{i}}_{j}$ for $j=$ $1, \ldots, M$. Note that the reduced system of equations can be built progressively, similar to the internal iterations in GMRES.

- Step 4. Add the result to the final solution, $\mathbf{I}_{\text {fin }}=\mathbf{I}_{\text {fin }}+\mathbf{I}$.

- Step 5. Compute the residue $\mathbf{r}=\mathbf{e}_{0}-\mathbf{Z I}_{\text {fin }}$.

- Step 6. Set the excitation vector e to the residue $r$ and go to Step 3 until the required residue is reached.

The main difference with GMRES is that the subspace is restarted on every subdomain.

\section{NUMERICAL RESULTS}

In this section the proposed approach is compared to the GMRES algorithm in terms of an error in surface current versus the solving complexity. The complexity is defined herein as the number of elementary operations " $a b+$ " (floating point product of complex scalar numbers and summation with another complex number), required to solve the problem, while the relative error in the surface current is computed as

$$
\epsilon=20 \log _{10}\left(\sqrt{\sum_{n}\left|I_{n}^{\text {approx }}-I_{n}^{\text {ref }}\right|^{2}} / \sqrt{\sum_{n}\left|I_{n}^{\text {ref }}\right|^{2}}\right),
$$

where $\mathbf{I}^{\text {approx }}$ is the current expansion coefficient vector, obtained using the proposed approach or restarted GMRES; and $\mathbf{I}^{\text {ref }}$ is the reference solution, obtained by direct solution of the MoM matrix equation (1).

The structures considered hereafter are subdivided into subdomains to have nearly equal and compact surfaces; for antenna arrays each subdomain is chosen to be a single antenna element. One possible way to improve the division into subdomains is through the so-called graph-partitioning technique (see e.g. [8]).

The system of equations (1) is assumed to be preconditioned for both CBFM and GMRES using the preconditioner described in [9], during which auxiliary subdomains are considered [7] in order to deal with the nearest interactions. Furthermore, a simplified version of GMRES is used [10], which implements the Gram-Schmidt orthogonalization of the vectors in the Krylov sub-space instead of using the Arnoldi iteration. This approach has a similar complexity as the original GMRES algorithm, while it is structurally closer to the CBFM.

For each geometry considered below a series of simulations have been performed for different numbers of CBFM"generating vectors" and different numbers of internal iterations (between restarts) for GMRES, and the best convergence curves of both methods are compared. Under "an iteration" for CBFM approach we understand hereafter a procedure consisting of (i) building the reduced system of equations of size $P M \times P M$, which involves $P$ mat-vecs, and (ii) solving this system.

Three geometries are considered:

- (Fig. 1) A sphere with radius $1.58 \lambda$, divided into $M=96$ or $M=384$ subdomains. The number of elementary basis functions (RWG) is $N=30720$. The sphere is excited by a plane wave.

- (Fig. 2) A rectangular plate with size $12 \lambda$, divided into $M=144$ or $M=256$ subdomains. The number of RWG basis functions is $N=42960$. The plate is excited by a plane wave under 45 degrees incidence.

- (Fig. 3) A 121-element dual-polarized array of both connected and disconnected Vivaldi tapered slot antennas. The numbers of RWG basis functions are $N=41975$ and $N=39325$ respectively. The array is uniformly excited by delta-gap voltage sources at each antenna element. The connected Vivaldi array has been designed by ASTRON [11]. 
Fig. 1 demonstrates the convergence rate of the newly defined iterative CBFM and GMRES for the sphere. If one aims at an accuracy in the surface current of e.g. $50 \mathrm{~dB}$, the domain-decomposition approach is more than twice faster than GMRES, i.e. with twice smaller operations count. The convergence in case of 96 subdomains is faster for both methods, and this can be explained by the influence of the preconditioner, which accounts for all adjacent neighbours of each subdomain. This is true as long as the solution time of the reduced system of equations is small compared to the matrixvector product needed to produce that system of equations. As explained in Section V, this supposes that the number of subdomains $M$ remains small compared to $F^{2}$, where $F>1$ is the DoF reduction factor ${ }^{2}$, which is satisfied in all numerical examples considered here.

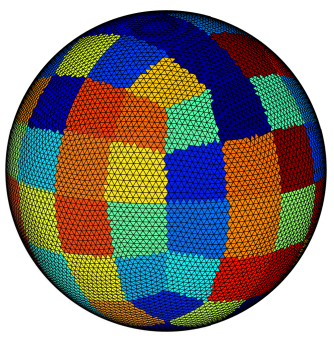

(a) 96 subdomains.

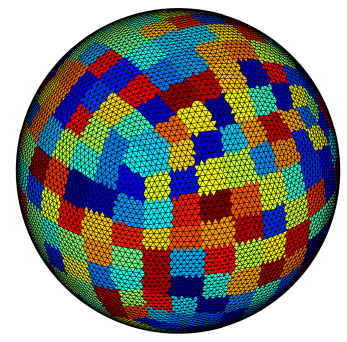

(b) 384 subdomains.

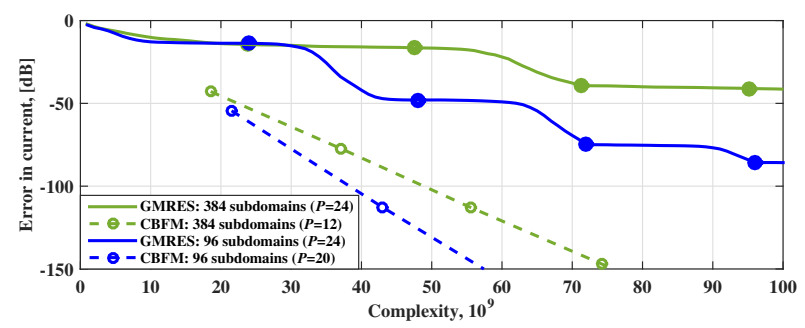

(c) GMRES and CBFM convergence.

Fig. 1. Numerical example 1: A sphere with radius $1.58 \lambda$, divided into (a) 96 subdomains and (b) 384 subdomains, and excited by an incident plane wave. Subfigure (c) compares the convergence rates of restarted GMRES and CBFM. The restart positions are indicated with circles.

Similar observations are made for the square plate shown in Fig. 2. However, the advantage of using the iterative CBFM is not significant in this case as compared to the more strongly coupled subdomains in the other examples.

The more complicated Vivaldi array case is demonstrated in Fig. 3. As expected, the disconnected array is a much easier case for numerical analysis, since no current can physically flow from one subdomain to another, which reduces mutual coupling, such that the convergence is much faster. The figure also shows that for the connected array the proposed domaindecomposition approach is more than a factor two faster, as compared to a conventional GMRES approach.

In all numerical examples the CBFM reaches an accuracy better than $-50 \mathrm{~dB}$ in only 1 to 2 iterations (for 0 to 1 restarts), with the number of mat-vecs per iteration equal to

${ }^{2} F=N_{s d} / P$ is average ratio between numbers of elementary basis functions and MBFs on each subdomain

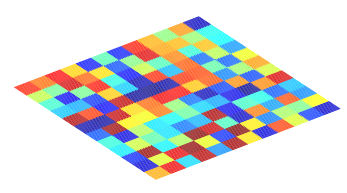

(a) 144 subdomains.

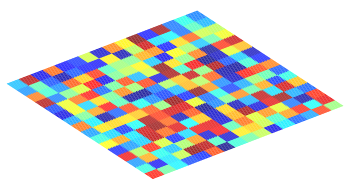

(b) 256 subdomains.

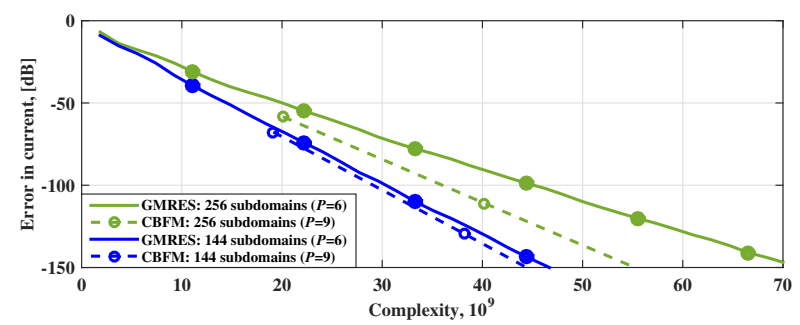

(c) GMRES and CBFM convergence.

Fig. 2. Numerical example 2: A rectangular plate with size $12 \lambda$, divided into (a) 144 subdomains and (b) 256 subdomains, and excited by a plane wave under $45 \mathrm{deg}$ incidence. Subfigure (c) compares the convergence rates of restarted GMRES and CBFM. The restart positions are indicated with circles.

$P$ as indicated in the legends of Figs. 1-3. GMRES requires 1 to 5 restarts to achieve similar accuracy levels.

It worth noting that we used an integral error in the surface current as a main figure of merit in this study. However, antenna characteristics, such as the antenna impedance and radiation pattern, are most commonly used by antenna designers. The relation between respective errors is not straightforward, however it can be assumed that the error in surface current and the error in antenna characteristics are of same order (see e.g. the approximation error analysis in [12], where different reflector antenna feeds are considered).

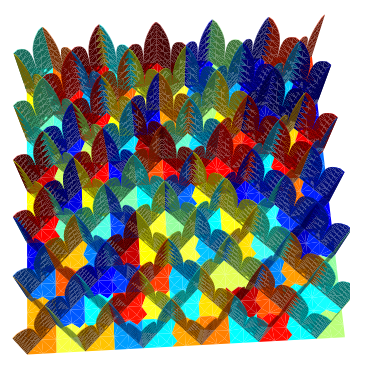

(a) connected array.

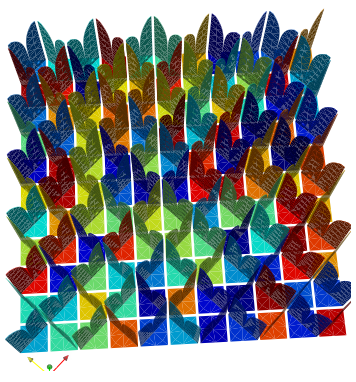

(b) disconnected array.

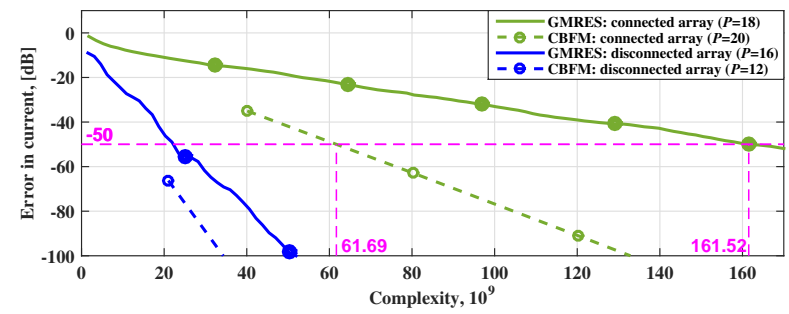

(c) GMRES and CBFM convergence.

Fig. 3. Numerical example 3: (a) A connected and (b) disconnected 121element dual-polarized Vivaldi array, divided into 121 subdomains, and excited by a delta-gap voltage sources at each antenna element. Subfigure (c) compares the convergence rates of restarted GMRES and CBFM. The restart positions are indicated with circles. 


\section{Discussion}

When well preconditioned, GMRES converges very rapidly (i.e. within a few tens of iterations), almost irrespective of the number of unknowns. As explained in [10], GMRES amounts to solving a reduced system of equations, whose size (i.e. number of DoFs), corresponds to the number of iterations. For large problems, this solution takes a negligible time as compared to that involved in the mat-vec operations. This means that, without significant increase in the computation time, one can afford more DoFs, as is the case with the approach proposed here, since the number of DoFs now corresponds to the number of mat-vecs $P$ multiplied by the number of subdomains. Without any specific matrix-vector multiplication, solving the reduced system of equations has a complexity $(P M)^{3}$ (here it is worth to mention that there are methods to reduce this exponent, see e.g. [13]), while the complexity of mat-vecs is $P N^{2}$. The increase of computational time is hence small as long as $P^{2} M \ll N_{s d}^{2}$, where $N_{s d}^{2}$ is the average number of elementary basis functions per subdomain.

It is pointed out that the gained accuracy does not seem to be commensurate with the increase of the degrees of freedom. More precisely, the achieved accuracy is not as good as that we may expect from GMRES when the number of iterations equals the total number of CBFs in the problem (in that case GMRES exploits the same number of DoF, at the expense of an excessive number of mat-vecs). This is probably due to the possible slight discontinuity between current distributions on contiguous subdomains; part of the newly generated DoFs may actually be needed to correct this deficiency.

In the very worst case, i.e., when the iterative CBF approach essentially provides the same accuracy as GMRES, one has two methods, one based on GMRES and one based on CBFs, with comparable accuracies when the number of iterations in the former is equal to the number of CBFs per subdomains in the latter. That equality is obtained by construction of the proposed method, since one new CBF per segment from the new generating vector is created at every iteration. It is interesting to notice that this equality precisely corresponds to the rule of thumb delineated from numerical experiments in [7] where MBFs (or CBFs) were created in a multiplescattering fashion, and it is shown here that this rule of thumb constitutes a lower bound for the capabilities of the iterative $\mathrm{CBF}$ (or MBF) approach.

It appears that a clear advantage beyond this rule is obtained with CBFs when - as proposed here - the CBFs on a given subdomain are simply taken as the segments of the generating vectors (which correspond to the subdomain of interest). Other (either purely algebraic or more physical) ways of creating the CBFs may allow us to further benefit from the larger number of DoFs created through the subdomain-based approach.

\section{CONCLUSIONS}

This work has introduced a domain-decomposition technique into Krylov subspace iteration, such as in GMRES for instance. This method is similar to the CBFM, here the MBFs are generated by simple segmentation of the pre-computed vectors of the Krylov subspace. The achieved convergence is faster than with GMRES by a factor ranging from 1.05 (the rectangular plate with large subdomains) to 2.6 (the connected Vivaldi array) while keeping the same accuracy. This opens new perspectives for the solution of multi-scaled radiation and scattering problems.

\section{APPENDIX}

To keep a well-conditioned reduced system of equations, the set $\mathbf{K}$ of MBFs should be orthogonalized by means of, e.g., a QR-decomposition. This slightly complicates the acceleration technique described in the Sec. II. The updated acceleration can be carried out in the following way.

Let us denote the orthogonalized matrix $\mathbf{K}$ as $\mathbf{K}^{\mathrm{o}}$, then (2b) becomes

$$
\tilde{\mathbf{Z}}_{i, j}=\mathbf{K}_{i}^{\mathrm{oH}} \mathbf{Z}_{i, j} \mathbf{K}_{j}^{\mathrm{o}} .
$$

After performing the QR-decomposition for each subdomain $j, \mathbf{K}_{j}=\mathbf{K}_{j}^{\mathrm{o}} \mathbf{R}_{j}$, Eq. (10) can be rewritten as

$$
\tilde{\mathbf{Z}}_{i, j}=\mathbf{K}_{i}^{\mathrm{o} H} \mathbf{Z}_{i, j} \mathbf{K}_{j} \mathbf{R}_{j}^{-1}=\mathbf{K}_{i}^{\mathrm{oH}} \mathbf{Q}_{i, j} \mathbf{R}_{j}^{-1},
$$

which only involves small matrices, and based on (7), is the final expression of the $(i, j)$ block of the reduced MoM matrix.

\section{REFERENCES}

[1] Y. Saad and M. H. Schultz, "GMRES: a generalized minimal residual algorithm for solving nonsymmetric linear systems," SIAM Journal on Scientific and Statistical Computing, vol. 7, no. 3, pp. 856-869, Jul. 1986.

[2] D. N. Wakam, J. Erhel, and W. D. Gropp, "Parallel adaptive deflated GMRES," in The 20th International Conference on Domain Decomposition Methods, UC San Diego, in La Jolla, California, Feb. 2011, pp. 631-638.

[3] E. Suter and J. R. Mosig, "A subdomain multilevel approach for the efficient MoM analysis of large planar antennas," Micr. Opt. Technol., vol. 26, no. 4, pp. 270-277, Aug. 2000.

[4] I. Stevanovic and J. R. Mosig, "Subdomain multilevel approach with fast MBF interactions," in Proc. IEEE AP-S International Symposium, Monterey, California, Jun. 2004, pp. 367-370.

[5] V. Prakash and R. Mittra, "Characteristic basis function method: A new technique for efficient solution of method of moments matrix equations," Micr. Opt. Technol., vol. 36, no. 2, pp. 95-100, Jan. 2003.

[6] L. Matekovits, G. Vecchi, G. Dassano, and M. Orefice, "Synthetic function analysis of large printed structures: the solution space sampling approach," in Proc. IEEE AP-S International Symposium, vol. 2, Boston, Massachusetts, Jul. 2001, pp. 568-571.

[7] N. Ozdemir, D. Gonzalez-Ovejero, and C. Craeye, "On the relationship between multiple-scattering Macro Basis Functions and Krylov subspace iterative methods," IEEE Trans. Antennas Propag., vol. 61, no. 4, pp. 2088-2098, Apr. 2013.

[8] R. Mitharwal and F. Andriulli, "On the multiplicative regularization of graph laplacians on closed and open structures with applications to spectral partitioning," IEEE Access, vol. 2, pp. 788-796, 2014.

[9] N. Ozdemir, D. Gonzalez-Ovejero, and C. Craeye, "A near-field preconditioner preserving the low-rank representation of method of moments interaction matrices," in Proc. Int. Conf. on Electromagn. in Adv. Applicat. (ICEAA), Torino, Italy, Sep. 2013, pp. 133-136.

[10] C. Craeye, J. Laviada, R. Maaskant, and R. Mittra, "Macro Basis Function framework for solving Maxwells equations in surface integral equation form," The FERMAT Journal, vol. 3, pp. 1-16, 2014.

[11] M. Arts, M. Ivashina, O. Iupikov, L. Bakker, and R. van den Brink, "Design of a low-loss low-noise tapered slot phased array feed for reflector antennas," in Proc. European Conference on Antennas and Propag. (EuCAP), Barcelona, Spain, Apr. 2010, pp. 1-5.

[12] O. A. Iupikov, R. Maaskant, M. Ivashina, A. Young, and P. Kildal, "Fast and accurate analysis of reflector antennas with phased array feeds including multiple reflections between feed and reflector," IEEE Trans. Antennas Propag., vol. 62, no. 7, Jul. 2014.

[13] A. Bojańczyk, "Complexity of solving linear systems in different models of computation," SIAM Journal on Numerical Analysis, vol. 21, no. 3, pp. 591-603, Jun. 1984. 